\title{
Heavy Rain-Producing Terrestrial Low Pressure Systems over the East Asian Summer Monsoon Region: Evolution, Energetics, and Trend
}

\author{
Yujia You, ${ }^{\mathrm{a}, \mathrm{b}}$ Mingfang Ting, ${ }^{\mathrm{a}}$ And Suzana J. CAmargo ${ }^{\mathrm{a}}$

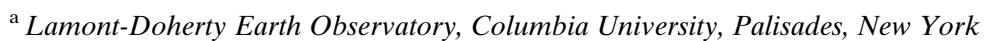 \\ ${ }^{\mathrm{b}}$ Department of Earth and Environmental Sciences, Columbia University, Palisades, New York
}

(Manuscript received 21 August 2020, in final form 29 December 2020)

\begin{abstract}
The synoptic low pressure systems (LPSs) formed over the downwind side of the Tibetan Plateau explain a substantial portion of summer rainfall extremes along their paths. Recent studies have found that the total extreme rainfall trend over the East Asian landmass, which features the "south flood-north drought" pattern, can be understood to a great extent by the changes in terrestrial LPSs. Yet, the energy sources fueling these storms and the environmental drivers of their long-term trends remain unclear. Utilizing a probabilistic clustering method, three clusters of terrestrial LPS tracks for the period 1979-2018 are identified. Besides the differences in trajectories that distinguish the clusters into northeastwardmigrating and quasi-stationary types, prominent intercluster differences are found in the LPS evolution, energetics, and trends. The Lorenz energetics suggest that while condensational heating is indispensable for all three clusters, the migratory type, which has greater intensity and faster development, is more closely tied to baroclinicity. Nonetheless, the summer baroclinicity alone is not enough to sustain these LPSs as these storms dissipate quickly after propagating out of the humid monsoon region and into the drier extratropics. Over time, occurrences of migratory LPSs decrease, and those of quasistationary LPSs increase. Using a Poisson model that links the LPS genesis to local environmental conditions, the decreasing occurrence of migratory LPSs is shown to result from the weakened baroclinicity, whereas the increasing occurrence of quasi-stationary LPSs is primarily driven by enhanced relative humidity and reduced steering flow in the mid-to-lower troposphere over East Asia.
\end{abstract}

KEYWORDS: Asia; Extratropical cyclones; Monsoons; Rainfall; Subtropical cyclones; Tropical cyclones

\section{Introduction}

Detecting and attributing the historical extreme rainfall trends over the densely populated East Asian monsoon region are of paramount scientific and socioeconomic importance. Previous observational studies have identified a statistically discernible dipole trend in summer extreme rainfall (MaySeptember) with decreasing trends over north-central China and increasing trends over southern China since the 1950s (e.g., Zhai et al. 2005; Liu et al. 2005; Dong et al. 2011; Ma et al. 2017). While this "south flood-north drought" pattern has been attributed to the weakened summer monsoon circulation (e.g., Wang and Zhou 2005) and the meridional shift of the East Asian jet stream (Day et al. 2018), several recent studies have attempted to provide new insights into the direct meteorological causes of the trend pattern by focusing on the excessive rain-generating synoptic low pressure systems (LPSs; Chang et al. 2012; Li et al. 2015; Zhang et al. 2018; You and Ting 2021).

The synoptic LPSs influencing East Asia originate in the western North Pacific Ocean in the form of tropical cyclones, as well as in the inland region downwind of the Tibetan Plateau in the form of terrestrial systems (e.g., Tao and Ding 1981; Hurley and Boos 2015). Even though these terrestrial LPSs often do not reach the same intensity as tropical cyclones, they account for a large portion of summertime torrential rainfall over inland areas as compared to tropical cyclones whose impacts

Corresponding author: Yujia You, yujia@ldeo.columbia.edu diminish rapidly toward the inland areas from the coast (Feng et al. 2016; Khouakhi et al. 2017; Guo et al. 2017; Zhang et al. 2018; You and Ting 2021). Indeed, the terrestrial LPStriggered rainfall, along with the consequential floods and landslides, has long plagued the lowlands and mountainous areas, particularly when these systems stall and have an ample supply of moisture (e.g., Tao and Ding 1981; Lu 1986). As reported by You and Ting (2021), the "south flood-north drought" extreme rainfall trend pattern is aligned with a decreasing number of northeastward-propagating terrestrial LPSs and a reduction of the average terrestrial LPS intensity (see their Fig. 6).

Despite the importance of terrestrial LPSs in driving the local extreme rainfall trends, their energy sources and the dynamical processes through which the large-scale environmental conditions modulate the long-term terrestrial LPS activity remain unclear. Research on terrestrial LPSs has a long history in the local meteorological community, where they have been referred to as the Jiang-Huai cyclone, Huang-Huai cyclone, southwest vortex, etc., based on their specific genesis locations. Nonetheless, most existing studies only consider the evolution of individual cases (e.g., Kuo et al. 1986; Wang and Orlanski 1987; Wang et al. 1993; Chang et al. 2000; Zhang and Zhao 2004; Chen et al. 2015), and the selected cases evolved in a variety of environmental conditions and exhibited a great diversity in their propagation, intensity, size, and duration. Thus it is difficult to understand the general characteristics of terrestrial LPS genesis and growth mechanisms. A large number of storms is therefore required to obtain a comprehensive understanding of the energetics of terrestrial LPSs. However, 
the observation-based track archive is not readily available for terrestrial LPSs due in part to the lack of universal identification criteria. Objective tracking algorithms and high-resolution reanalyses were, therefore, employed by several recent climatological studies (Feng et al. 2016; You and Ting 2021). Specifically, You and Ting (2021) suggested that terrestrial LPSs share some properties with both tropical and extratropical cyclones and likely belong to a hybrid storm type, as indicated by the baroclinic and warm-core structure seen in the cyclone centered composites. Yet, the complete energy budget of terrestrial LPSs has not yet been explored.

The primary purpose of the present study is to investigate the dynamical processes fueling the various types of terrestrial LPSs and to explore the environmental drivers of their trends. Here we utilize a large number of reanalysis-derived terrestrial LPS trajectories compiled using an objective feature-tracking algorithm (You and Ting 2021) and carry out a probabilistic cluster analysis to classify terrestrial LPSs into a few clusters, so that the genesis location, propagation, intensity, and evolution characteristics of LPSs are stratified by cluster. This approach also allows us to consider the observed terrestrial LPS trends as changes in the occurrence of different clusters. Evaluating the energy sources and environmental drivers of the different terrestrial LPS clusters could yield valuable information about the ultimate causes of observed extreme rainfall trends over the past decades and provide useful insights into the possible future changes under anthropogenic climate change.

The remainder of this paper is organized as follows. Section 2 presents the data and method used in this study. In section 3, we investigate several properties of terrestrial LPSs in each cluster, including track, temporal evolution, intensity, and rainfall characteristics. The energetics of terrestrial LPS clusters as well as the association between the trend of cluster occurrence and changes in environmental fields are also examined. The main results are summarized in section 4 .

\section{Data and methods}

\section{a. Data and LPS tracking method}

The Asian monsoon LPS trajectories employed here are taken from those compiled in You and Ting (2021), where a detailed description of the objective feature-tracking algorithm is provided. Briefly stated, the tracking algorithm was applied to the 6-hourly 850-hPa relative vorticity outputs from the European Centre for Medium-Range Weather Forecasts interim reanalysis dataset (ERA-Interim, herein ERA-I), which spans the period 1979-2018 (Dee et al. 2011). The relative vorticity was spectrally smoothed to retain total wavenumbers 5-42 before tracking, and the local maxima greater than the threshold value of $5 \times 10^{-6} \mathrm{~s}^{-1}$ at consecutive time steps were tracked and connected to form initial trajectories (Hodges 1994, 1999). Only storms lasting longer than 2 days, spending at least one day in the East Asian monsoon region, formed over land grid cells, and accompanied by a minimum sea level pressure (SLP) anomaly relative to a 21-day running mean are considered. Note that the systems moving off from the Tibetan Plateau, which are most prominent at $500 \mathrm{hPa}$ and can occasionally trigger the genesis of terrestrial LPSs or strengthen pre-existing LPSs, are not taken into account in the present study (e.g., Curio et al. 2019).

To measure the LPS activity, the LPS genesis (track) density is computed by counting the number of LPS genesis (track) points within a $250-\mathrm{km}$ radius of each grid point. The translation speed of LPS at each grid point is derived using neighboring track positions at 6-hourly intervals along each trajectory. The 6-hourly and monthly atmospheric fields from the ERA-I are utilized to examine the association between the terrestrial LPSs and environmental conditions. The short-range rainfall accumulation forecast from ERA-I is used because the gauge-based daily rainfall observation lacks data over open oceans. To measure convective instability, we calculate equivalent potential temperature using the formulation suggested by Bolton (1980). The ERA-I data used here are on a $0.75^{\circ} \times 0.75^{\circ}$ horizontal grid and are available on 27 vertical pressure levels spacing nonuniformly from 1000 to $100 \mathrm{hPa}$.

\section{b. Clustering method}

The probabilistic clustering technique developed by Gaffney (2004) is used to categorize terrestrial LPS trajectories into a certain number of clusters with similar tracks. Using the latitudinal and longitudinal positions of individual LPSs as inputs, the technique builds a mixture of polynomial regression curves (quadratic here) to fit the geographical shape of the trajectories by maximizing the likelihood of the regression parameters. Although higher-order polynomials could be used, previous studies have indicated that the quadratic polynomial regression functions appear to offer the best trade-off between ease of interpretation and goodness of fit (e.g., Gaffney et al. 2007; Camargo et al. 2007a). As a "hard" clustering method, each trajectory is assigned to one of the clusters, which has its own unique shape parameters and mean regression track. For any cluster, the mean regression track represents the polynomial regression curve that best fits all the trajectories in that cluster. To avoid subjectivity in assigning each trajectory to a specific cluster, we use a Monte Carlo technique following Camargo et al. (2007a): the cluster analysis is repeated 500 times by randomizing the order of trajectories included in the analysis. The final cluster assignment is the one with the largest likelihood value among the 500 trials. The ordering of the resulting clusters is random and does not indicate the importance of each cluster. Compared to the $k$-means clustering, this clustering technique can handle variable-length trajectories without converting them to a predefined coordinate of equal length, such as the lifetime percentage axis. This probabilistic clustering technique has been extensively applied to observed tropical cyclone tracks in various ocean basins (Camargo et al. 2007a,b, 2008; Kossin et al. 2010; Ramsay et al. 2012), the North Atlantic extratropical cyclone tracks (Gaffney et al. 2007), and tropical cyclone tracks in various climate models (Daloz et al. 2015; Nakamura et al. 2017; Patricola et al. 2018; Ramsay et al. 2018).

\section{c. Lorenz energy cycle}

To uncover the energetics of LPSs, the limited-area Lorenz energy cycle, which examines the generation and conversion of 

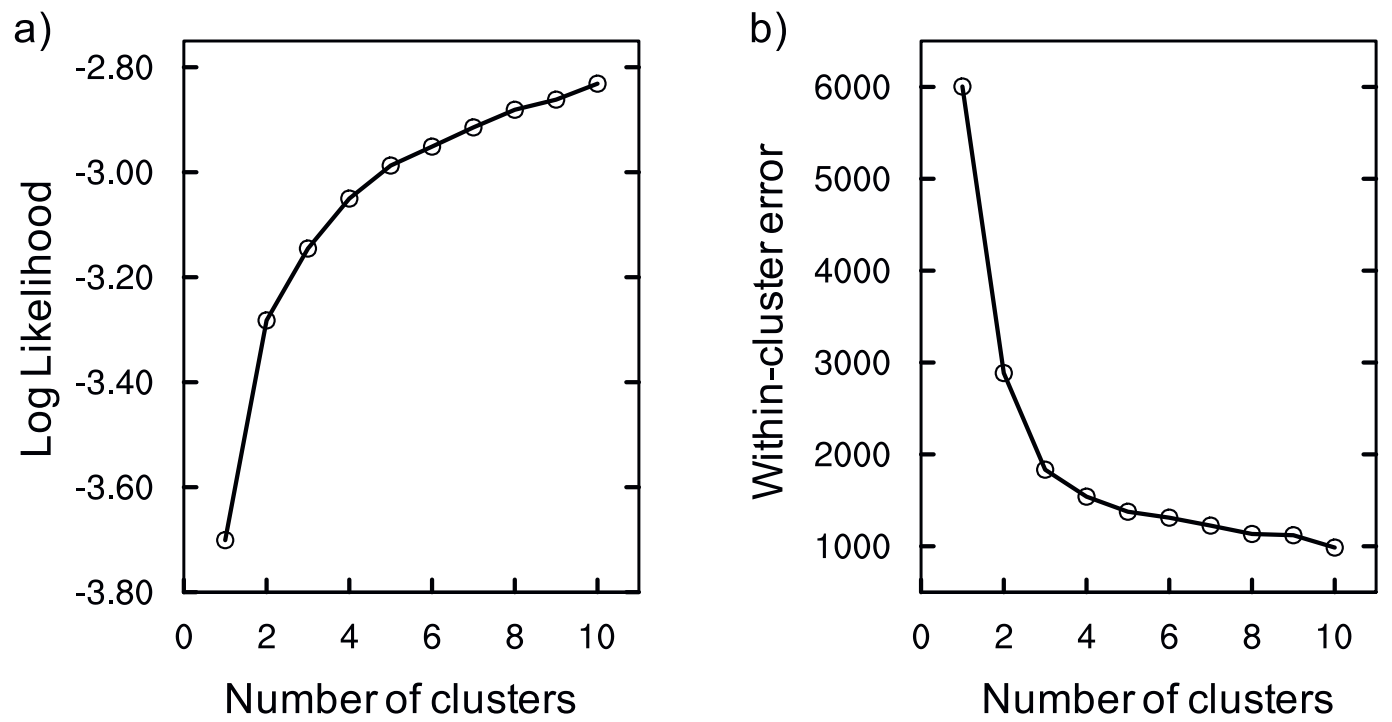

FIG. 1. (a) Log-likelihood values and (b) within-cluster spreads as a function of the number of clusters.

the various forms of energy in a straightforward way, is adopted (e.g., Lorenz 1955). The energy budget equations for the zonal-mean and eddy components of the available potential energy $\left(A_{Z}\right.$ and $\left.A_{E}\right)$ and kinetic energy $\left(K_{Z}\right.$ and $\left.K_{E}\right)$ can be written as follows:

$$
\begin{aligned}
& \frac{\partial A_{z}}{\partial t}=-C_{Z}-C_{A}+G_{Z}+B A_{Z}, \\
& \frac{\partial K_{z}}{\partial t}=C_{Z}-C_{K}-D_{Z}+B K_{Z}+B \Phi_{Z}, \\
& \frac{\partial A_{E}}{\partial t}=C_{A}-C_{E}+G_{E}+B A_{E}, \\
& \frac{\partial K_{E}}{\partial t}=C_{E}+C_{K}-D_{E}+B K_{E}+B \Phi_{E},
\end{aligned}
$$

where $C_{Z}$ denotes the energy conversion from $A_{Z}$ to $K_{Z}, C_{A}$ from $A_{Z}$ to $A_{E}, C_{K}$ from $K_{Z}$ to $K_{E}$, and $C_{E}$ from $A_{E}$ to $K_{E}$. Additionally, $G_{Z}$ and $G_{E}$ represent the generations of $A_{Z}$ and $A_{E}$ by diabatic processes, and $D_{Z}$ and $D_{E}$ are the dissipation of $K_{Z}$ and $K_{E}$ due to friction. The limited-area energy cycle is closed by energy transport $\left(B A_{Z}, B A_{E}, B K_{Z}, B K_{E}\right)$ and pressure work $\left(B \Phi_{Z}, B \Phi_{E}\right)$ through the lateral boundaries. The complete mathematical formulas for each of the terms can be found in Michaelides (1987, 1992), Michaelides et al. (1999), and Veiga and Ambrizzi (2013), and references therein.

Here we conduct the limited-area Lorenz energetics analysis in a quasi-Lagrangian framework, in which the computational domain always encompasses the LPS being studied. At each time step, the energy budget is performed in a $20^{\circ} \times 20^{\circ}$ latitude/longitude atmospheric cube centered at the LPS and bounded by $1000-$ and $100-\mathrm{hPa}$ pressure levels in the vertical. The results presented are insensitive to reasonable choices of the domain size (i.e., $20^{\circ}-30^{\circ}$ ). Compared to the traditional Eulerian scheme using a geographically fixed computational domain, the quasi-Lagrangian framework shows significant advantages for studying mobile systems and for better representing the cyclone's energetics as it focuses on the target system (e.g., Michaelides et al. 1999). The quasi-Lagrangian framework brings some difficulties in computing the time tendency terms owing to the horizontal displacement of the computational domain. In this study we focus primarily on the energy conversion and generation terms and use the ERA-I 6-hourly fields for calculating the energetics. The fourdimensional diabatic heating used to compute the diabatic energy generation term is diagnosed as a residual from the thermodynamic equation (e.g., Chan and Nigam 2009).

\section{Results}

\section{a. Properties of terrestrial LPS clusters}

One common challenge for any clustering technique is the optimal choice of the number of clusters. As in Camargo et al. (2007a), we determine the most appropriate number of clusters based on two criteria: the in-sample log-likelihood and the within-cluster spread. The log-likelihood is the natural logarithm of the likelihood, measuring how well the polynomial regression curves fit the trajectories. The within-cluster spread denotes the difference of tracks in latitude and longitude from the mean regression track, squared and summed over all tracks in the cluster. As shown in Fig. 1, the log-likelihood value increases and within-cluster spread decreases with increasing number of clusters, indicating an improvement in fit. In terms of both metrics, there are consistent improvements as the number of clusters increases. However, both exhibit a flattening curve beyond three clusters, indicating gradually diminishing improvements when more clusters are added. This is particularly the case for the within-cluster spread. Hence, three clusters were chosen in this study. 
TABLE 1. Statistics of clusters: number of LPSs, percentage of total (\%), genesis position (latitude, longitude), peak intensity location (latitude, longitude), lifetime (days), translation vector (zonal, meridional components; $\mathrm{km} \mathrm{h}^{-1}$ ), and lifetime maximum intensity $\left(10^{-5} \mathrm{~s}^{-1}\right)$. Values inside the parentheses are standard deviations. An asterisk $\left(^{*}\right)$ indicates that the cluster's mean statistics are significantly different from that of the cluster 2 LPSs based on a nonparametric bootstrapping method.

\begin{tabular}{|c|c|c|c|}
\hline & Cluster 1 & Cluster 2 & Cluster 3 \\
\hline No. of LPSs in 40 years & 181 & 186 & 264 \\
\hline Percentage of total $(\%)$ & 29 & 29 & 42 \\
\hline Mean genesis position (lat, lon) & $31^{\circ} \mathrm{N}\left( \pm 3.5^{\circ}\right), 111^{\circ} \mathrm{E}\left( \pm 4.1^{\circ}\right)^{*}$ & $27^{\circ} \mathrm{N}\left( \pm 3.4^{\circ}\right), 107^{\circ} \mathrm{E}\left( \pm 3.9^{\circ}\right)$ & $25^{\circ} \mathrm{N}\left( \pm 3.3^{\circ}\right), 105^{\circ} \mathrm{E}\left( \pm 2.6^{\circ}\right)^{*}$ \\
\hline Median genesis position (lat, lon) & $30^{\circ} \mathrm{N}, 112^{\circ} \mathrm{E}$ & $27^{\circ} \mathrm{N}, 107^{\circ} \mathrm{E}$ & $25^{\circ} \mathrm{N}, 105^{\circ} \mathrm{E}$ \\
\hline Mean peak intensity position (lat, lon) & $37^{\circ} \mathrm{N}\left( \pm 5.2^{\circ}\right), 133^{\circ} \mathrm{E}\left( \pm 16.5^{\circ}\right)^{*}$ & $33^{\circ} \mathrm{N}\left( \pm 4.7^{\circ}\right), 124^{\circ} \mathrm{E}\left( \pm 14.5^{\circ}\right)$ & $25^{\circ} \mathrm{N}\left( \pm 3.9^{\circ}\right), 107^{\circ} \mathrm{E}\left( \pm 4.2^{\circ}\right)^{*}$ \\
\hline Median peak intensity position (lat, lon) & $36^{\circ} \mathrm{N}, 126^{\circ} \mathrm{E}$ & $32^{\circ} \mathrm{N}, 122^{\circ} \mathrm{E}$ & $26^{\circ} \mathrm{N}, 107^{\circ} \mathrm{E}$ \\
\hline Mean lifetime (days) & $7( \pm 3.5)^{*}$ & $5.6( \pm 2.9)$ & $4.9( \pm 2.5)^{*}$ \\
\hline Median lifetime (days) & 6.2 & 4.7 & 4.5 \\
\hline $\begin{array}{l}\text { Mean translation vector (zonal, } \\
\text { meridional; } \mathrm{km} \mathrm{h}^{-1} \text { ) }\end{array}$ & $25.2( \pm 10.3), 7.8( \pm 6.2)$ & $14.9( \pm 7.8), 4.7( \pm 4.8)$ & $3.8( \pm 6.8), 0.28( \pm 5.3)$ \\
\hline $\begin{array}{l}\text { Median translation vector (zonal, } \\
\text { meridional; } \mathrm{km} \mathrm{h}^{-1} \text { ) }\end{array}$ & $24.7,7.4$ & $14.8,4.8$ & $2.7,0.31$ \\
\hline $\begin{array}{l}\text { Mean lifetime maximum intensity } \\
\left(10^{-5} \mathrm{~s}^{-1}\right)\end{array}$ & $9.1( \pm 3.8) *$ & $7.1( \pm 3.1)^{*}$ & $4.2( \pm 1.3)^{*}$ \\
\hline $\begin{array}{l}\text { Median lifetime maximum intensity } \\
\quad\left(10^{-5} \mathrm{~s}^{-1}\right)\end{array}$ & 8.2 & 6.7 & 4.1 \\
\hline
\end{tabular}

The general characteristics for each of the three clusters are summarized in Table 1. Of the 631 LPSs during the 40-yr period, $\sim 29 \%$ are assigned to cluster $1, \sim 29 \%$ to cluster 2 , and $\sim 42 \%$ to cluster 3 . The climatological genesis density, translation velocity, and track density are presented in Fig. 2 for each of the three clusters, along with all the storm trajectories and the mean regression tracks. While the mean genesis positions shift southwestward from cluster 1 to cluster 3, the LPSs in all three clusters tend to form over the downwind flank of the Tibetan Plateau (gray shadings), suggesting the effect of terrain on cyclogenesis (Figs. 2a,d,g). The mean genesis latitude and longitude of the three clusters are significantly different at a $95 \%$ confidence level based on a nonparametric bootstrapping method. More pronounced differences among clusters are found in the translation speed and the length of trajectories, which clearly divide the three clusters into two distinct regimes, namely the migratory (Figs. 2b,e) and the quasi-stationary types (Fig. 2h). In contrast to clusters 1 and 2, in which the LPSs exhibit a considerable degree of mobility and migrate northeastward toward the midlatitude North Pacific, the cluster-3 LPSs are almost stagnant with a translation speed of $\sim 4 \mathrm{~km} \mathrm{~h}^{-1}$. The mean translation speed for cluster-1 LPSs $\left(\sim 27 \mathrm{~km} \mathrm{~h}^{-1}\right)$ is faster than that of the cluster-2 LPSs $\left(\sim 16 \mathrm{~km} \mathrm{~h}^{-1}\right)$. The difference in translation speed primarily reflects the difference in ambient steering winds and is not related to the genesis locations of the three clusters (as shown later in Fig. 7).

Besides the differences in translation velocity that distinguish the three clusters, pronounced differences are noted in mean peak intensity location, lifetime maximum intensity, and timing of rainfall. Here the LPS intensity is defined as the relative vorticity at the LPS center. Figure 3 presents the maximum intensity locations of the LPSs for each of the cluster and the composites of daily anomalous precipitation during all LPS active days. The anomaly is calculated relative to the $40-\mathrm{yr}$ daily climatology. On average, the lifetime maximum intensity is the highest for cluster $1\left(\sim 9 \times 10^{-5} \mathrm{~s}^{-1}\right)$ and decreases to $\sim 7 \times 10^{-5} \mathrm{~s}^{-1}$ for cluster 2 and $\sim 4 \times 10^{-5} \mathrm{~s}^{-1}$ for cluster 3 (Table 1 ). The migratory clusters reach peak intensity near the northeast coasts of China, the Korean Peninsula, and Japan (Figs. 3a,b), whereas the quasi-stationary LPSs in cluster 3 achieve the peak intensity slightly east of the storm genesis locations (Fig. 3c). The time of the peak intensity separates the life cycle of LPSs into developing and decaying phases corresponding to the period before and after the peak intensity, respectively. For migratory clusters, the most intense rainfall takes place prior to the maximum intensity along the LPS trajectories (Figs. 3d,e), whereas this relationship is not evident in the quasi-stationary cluster (Fig. 3f). In all three clusters, precipitation gets suppressed over the South and East China Seas, indicating moisture divergence in these regions as moist air over these areas is drawn into the LPSs.

To better illustrate the connection between storm intensity and rainfall, we composite the intensity and associated rainfall with respect to each LPS's lifetime maximum intensity (Fig. 4, left column). The LPS-related rainfall is measured by the areaaveraged rainfall within 500-km radius from the LPS center, as in You and Ting (2021). As a reference, the composited minimum SLP anomaly relative to a 21-day running mean for each LPS is also shown with respect to the lifetime vorticity maximum (Fig. 4, right column). The migratory LPSs in clusters 1 and 2 exhibit similar characteristics, with the most intense rainfall rate occurring during the developing stage and declining rapidly after the peak intensity (Figs. 4a-d). In contrast, for the quasi-stationary LPSs in cluster 3, the most intense rainfall tends to occur synchronously with the maximum storm intensity (Figs. 4e,f).

Noticeable intercluster differences also exist in the trends of genesis frequency and LPS-related rainfall. Figure 5 depicts the linear trends of genesis density, track density, and LPSrelated summer rainfall totals for each of the clusters. The 
Genesis density
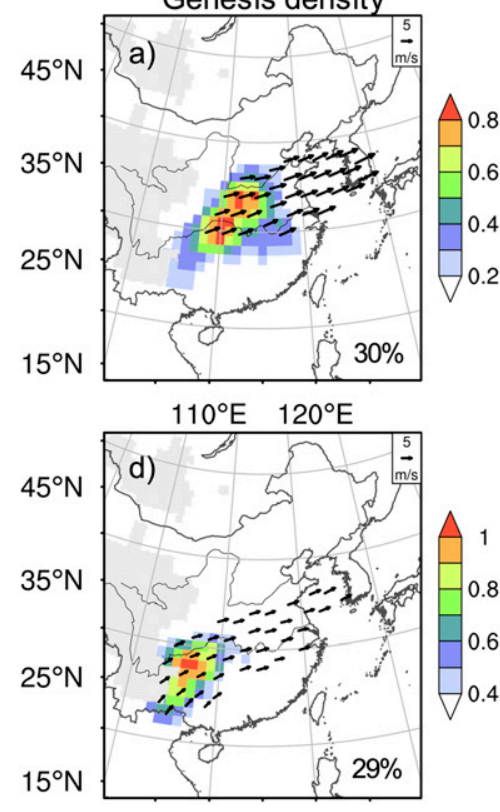

$110^{\circ} \mathrm{E} \quad 120^{\circ} \mathrm{E}$

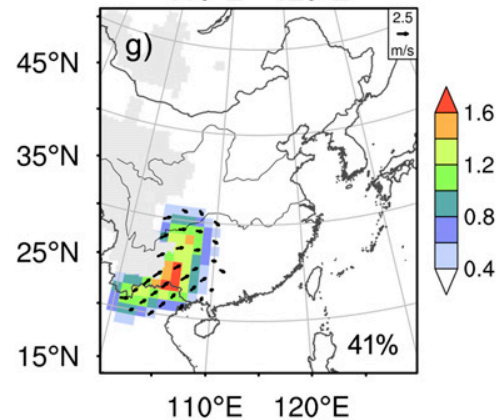

Track density

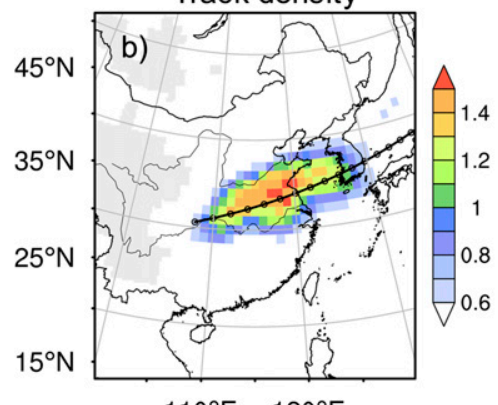

$110^{\circ} \mathrm{E} \quad 120^{\circ} \mathrm{E}$

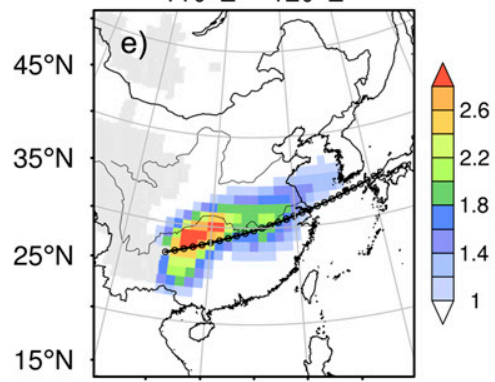

$110^{\circ} \mathrm{E} \quad 120^{\circ} \mathrm{E}$

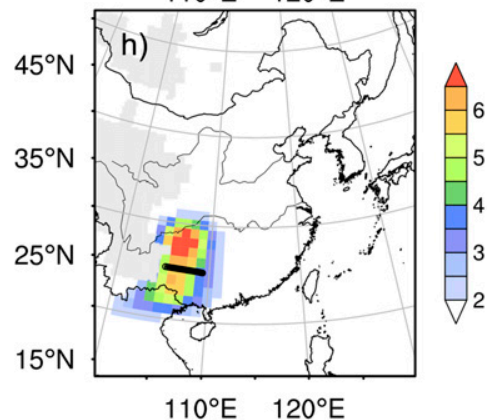

Tracks
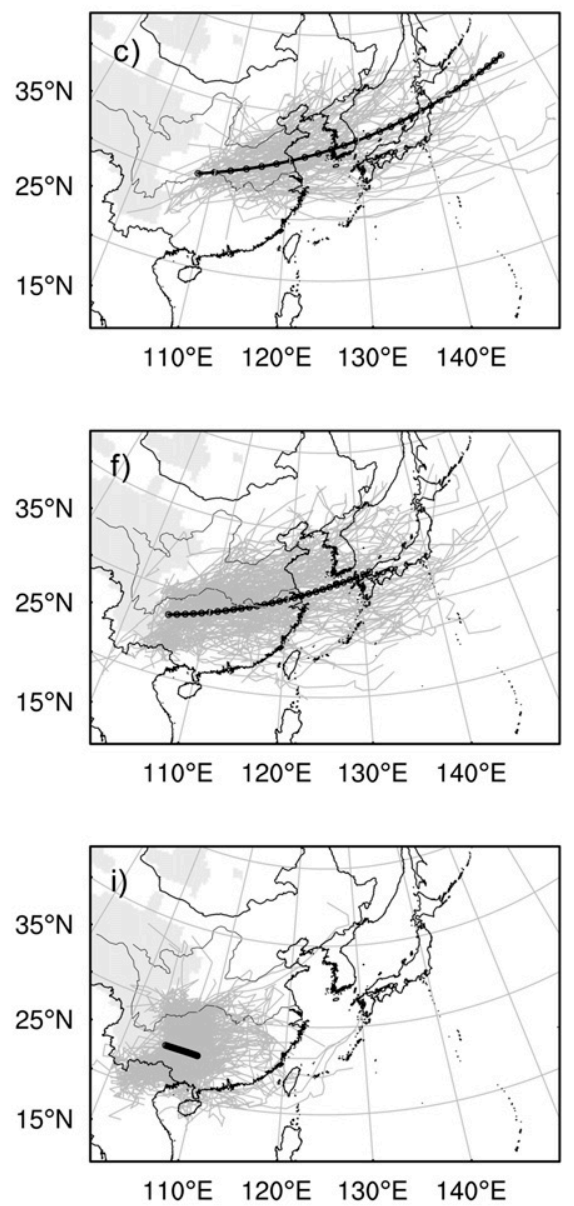

FIG. 2. Climatological (a),(d),(g) genesis density (shading; number of LPSs per summer) and translation velocity (vector; m $\mathrm{s}^{-1}$ ), (b),(e),(h) track density (shading; number of LPS days per summer) and mean regression trajectory (black curves), and (c),(f),(i) all LPS tracks (gray curves) and the mean regression trajectories (black curves) for (top) cluster 1, (middle) cluster 2, and (bottom) cluster 3. The gray shading denotes regions where the 850 -hPa pressure surface falls below ground.

Mann-Kendall test is employed to evaluate the statistical significance of the monotonic trends. Toward the end of the study period, the migratory (quasi-stationary) LPSs in clusters 1 and 2 (cluster 3 ) occur less (more) often (Figs. 5a-c), leading to a grossly reduced (increased) track density everywhere (Figs. 5d-f) and a statistically significant decline (increase) in LPS-related rainfall (Figs. 5d-i). Collectively, these trends contribute to the "north drought-south flood" rainfall trend pattern as shown in You and Ting (2021). As the total number of terrestrial LPSs does not exhibit a statistically significant trend (not shown), the trends in different clusters imply that terrestrial LPSs may have experienced a regime shift toward less migratory systems and more quasi-stationary systems during the study period.

\section{b. Energetics of terrestrial LPS clusters}

The regime shift shown in Fig. 5 implies that the migratory and staggering LPSs are favored by different environmental conditions. While this regime shift could be partially induced by changes in steering flow as hinted by the intercluster difference in translation speed, the prominent difference in the relationship between LPS intensity and rainfall (Fig. 4) indicates that the generation and intensification mechanisms of migratory and quasi-stationary clusters may be different. To what extent are the three terrestrial LPS clusters fueled by diabatic processes associated with latent heat release as in tropical cyclones versus by baroclinic instability as in midlatitude cyclones? To address this question, we calculate the Lorenz energy cycle for each cluster. Similar composites as in Fig. 4 are carried out for the relevant energy conversion terms and the diabatic generation of eddy available potential energy term, as depicted in Fig. 6.

For all three clusters, the barotropic conversion between zonal mean kinetic energy and eddy kinetic energy $\left(C_{K}\right)$ has a negligible amplitude and barely exhibits any temporal fluctuations (purple lines), indicating that very little kinetic energy of 

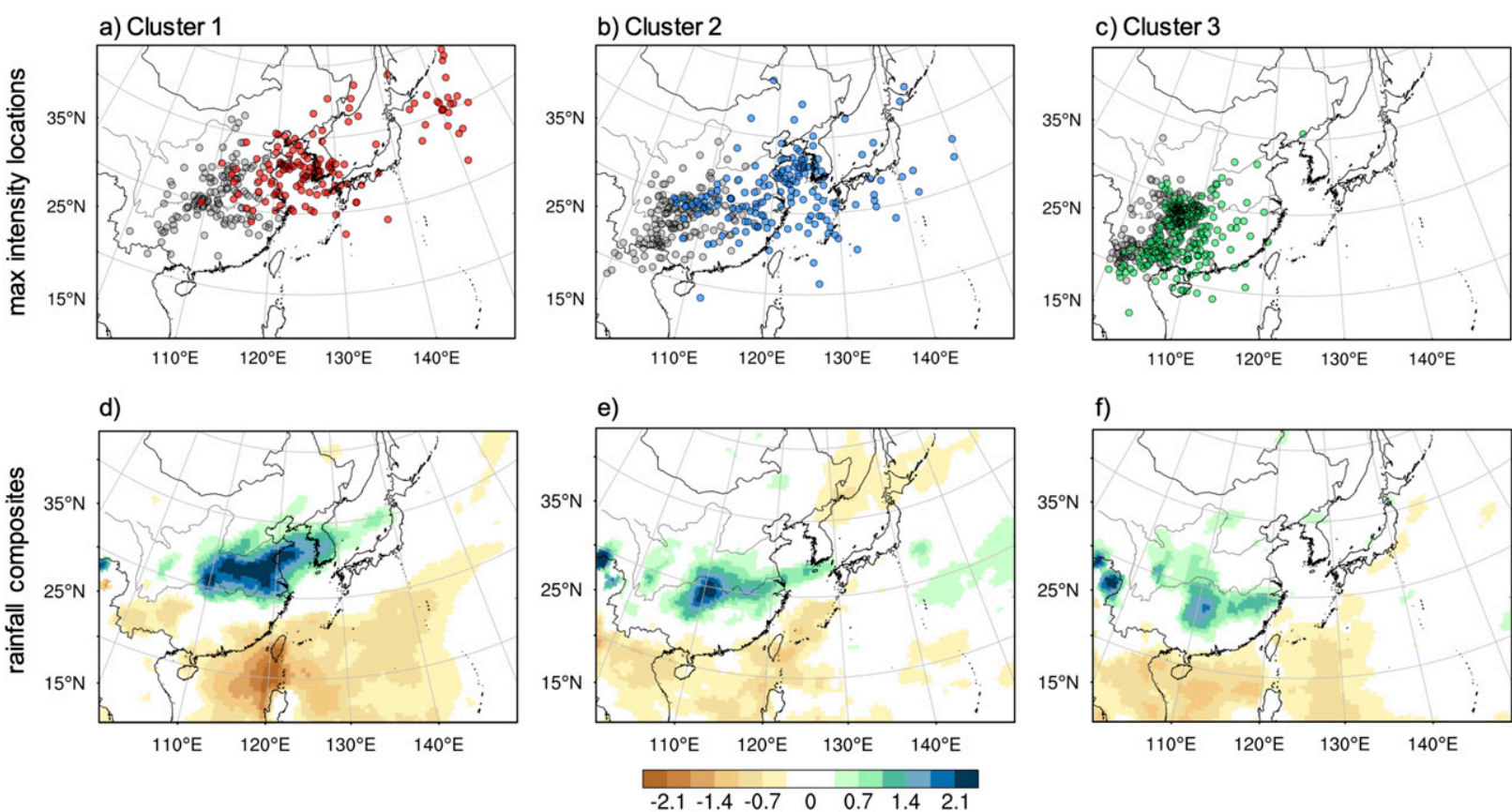

FIG. 3. (a)-(c) Geographical distribution of formation (gray dots) and lifetime maximum intensity locations (colored dots) of LPSs in different clusters, and (d)-(f) composite of anomalous daily precipitation (shading; $\mathrm{mm} \mathrm{day}^{-1}$ ) with respect to the 40-yr climatology for (left) cluster 1, (center) cluster 2, and (right) cluster 3 . In (d)-(f), only values significant at the $95 \%$ confidence level based on a two-tailed Monte Carlo bootstrapping test are shown.

the zonal-mean flow is extracted by the eddies. The eddy kinetic energy $K_{E}$ is mostly converted from the available potential energy $A_{E}$ through baroclinic processes $C_{E}$ (red lines). The available potential energy $A_{E}$, in turn, is generated from both the diabatic process $\left(G_{E}\right.$, green lines) and is converted from the zonal available potential energy $\left(C_{A}\right.$, cyan lines). Additionally, the evolution of $C_{E}$ is in general agreement with $C_{A}$ and $G_{E}$, highlighting the contribution from both the baroclinic and diabatic processes in the maintenance of cyclone intensity. The predominance of the two terms corroborates
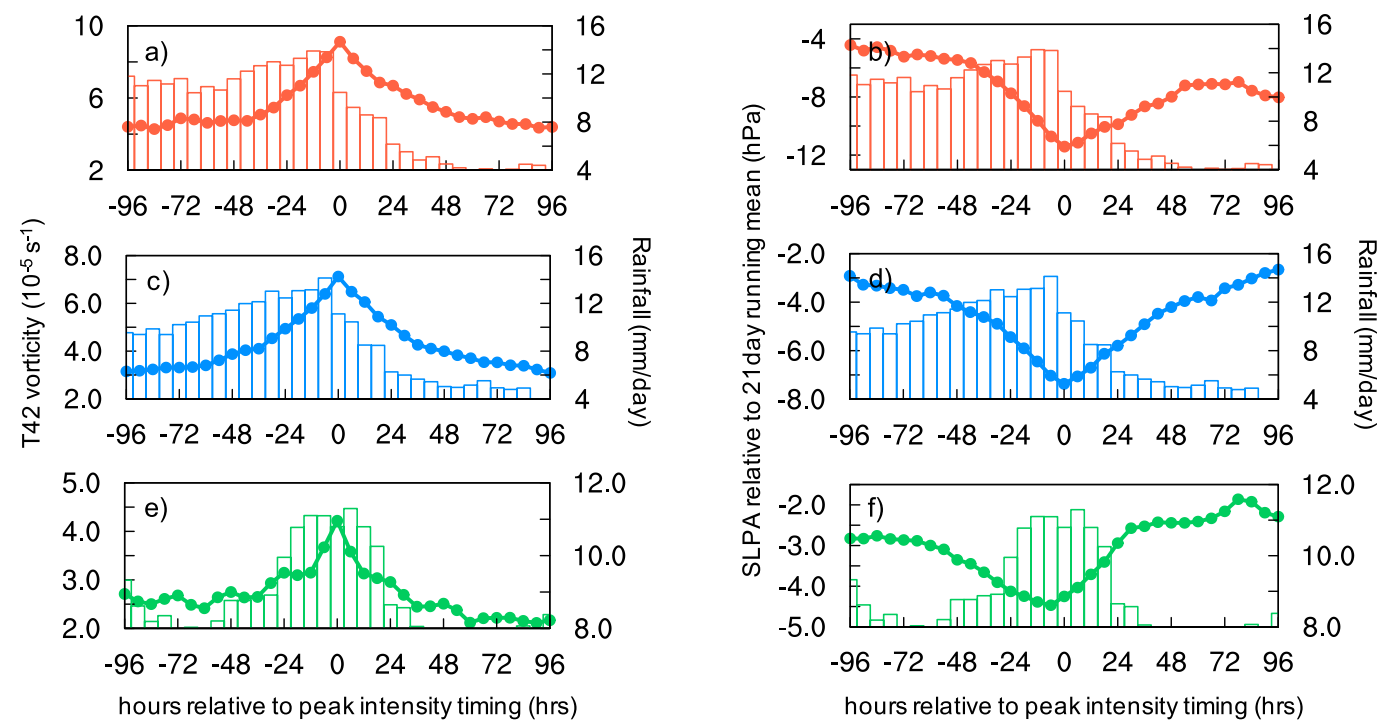

FIG. 4. Quasi-Lagrangian composites of the temporal evolution with respect to the maximum LPS intensity of each individual cluster for (a),(c),(e) relative vorticity (solid lines; $10^{-5} \mathrm{~s}^{-1}$ ) and (b),(d),(f) sea level pressure anomalies from the 21-day running mean (solid lines; hPa) for (top) cluster 1, (middle) cluster 2, and (bottom) cluster 3. Also shown in each panel are the composites of LPS-related rainfall (colored bars; $\mathrm{mm} \mathrm{day}^{-1}$ ) for the respective cluster. 

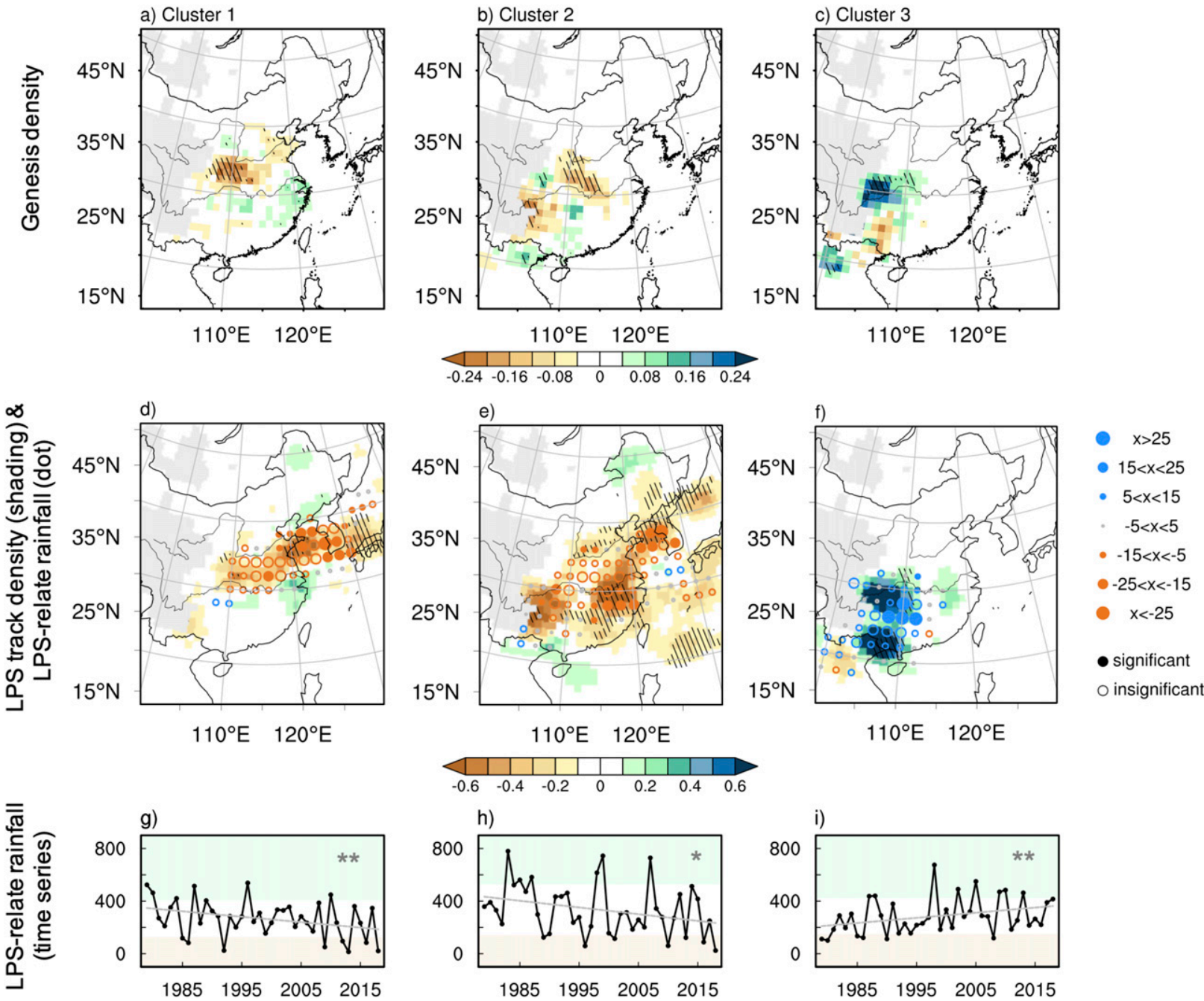

FIG. 5. (a)-(c) Linear trend of genesis density (number of LPSs per decade; shading), (d)-(f) linear trend of track density (number of LPS days per decade; shading) and LPS-related rainfall ( $\mathrm{mm} \mathrm{decade}^{-1}$; dots), and (g)-(i) yearly time series of the LPS-related summer rainfall totals ( $\mathrm{mm}$; black line) for (left) cluster 1, (center) cluster 2, and (right) cluster 3. The stippling in (a)-(f) indicates statistical significance greater than $90 \%$ confidence level according to a nonparametric Mann-Kendall test. In (g)-(i), the gray lines represent the least squares fitted trends, the green (brown) shadings denote values greater (less) than one standard deviation, and the single and double asterisks indicate trends significant at the $90 \%$ and $95 \%$ confidence level, respectively, according to a nonparametric Mann-Kendall test.

that both latent heat release and baroclinic instability are indispensable sources for the development of terrestrial LPSs. This finding is consistent with existing theories of the moist baroclinic instability, which fosters the intensification, promotes updrafts, and facilitates faster cyclone development (e.g., Mak 1982; Gutowski et al. 1992; Krishnamurti et al. 2013; Cohen and Boos 2016).

Among LPS clusters and between the developing and decaying stages of LPS, the relative importance of $C_{A}$ and $G_{E}$ varies markedly. For the weaker quasi-stationary LPSs in cluster $3, G_{E}$ dominates over $C_{A}$ throughout the life cycle (Fig. 6d), indicating that latent heat rather than baroclinicity is the main cause of the cluster-3 storm intensification. This explains why the peak intensity occurs simultaneously with the maximum rainfall as shown in Figs. 4e and 4f. For the migratory LPSs in clusters 1 and 2, in contrast, $C_{A}$ and $G_{E}$ are of equal importance during the developing stage, while $G_{E}$ appears to be the primary factor controlling the decaying of storms since it declines more rapidly than $C_{A}$ after the peak intensity (Figs. 6b,c). This agrees well with the quick reduction of rainfall before peak strength of migratory LPSs as shown in Figs. 4a-d, which suggests that even though the migratory LPSs propagate northeastward into the summer subtropical jet, they are not able to sustain peak intensity without latent heat release. As the rainfall is determined jointly by moisture supply and storm-induced convergence, we speculate that the distinct behavior of migratory and quasi-stationary clusters might result from the different environmental factors constraining their development. Specifically, the decay of the migratory cluster is likely owing to the lack of environmental moisture supply, while the cluster-3 LPSs are prohibited from 


$-\mathrm{C}_{\mathrm{K}}-\mathrm{C}_{\mathrm{E}}-\mathrm{C}_{\mathrm{A}}-\mathrm{G}_{\mathrm{E}}$
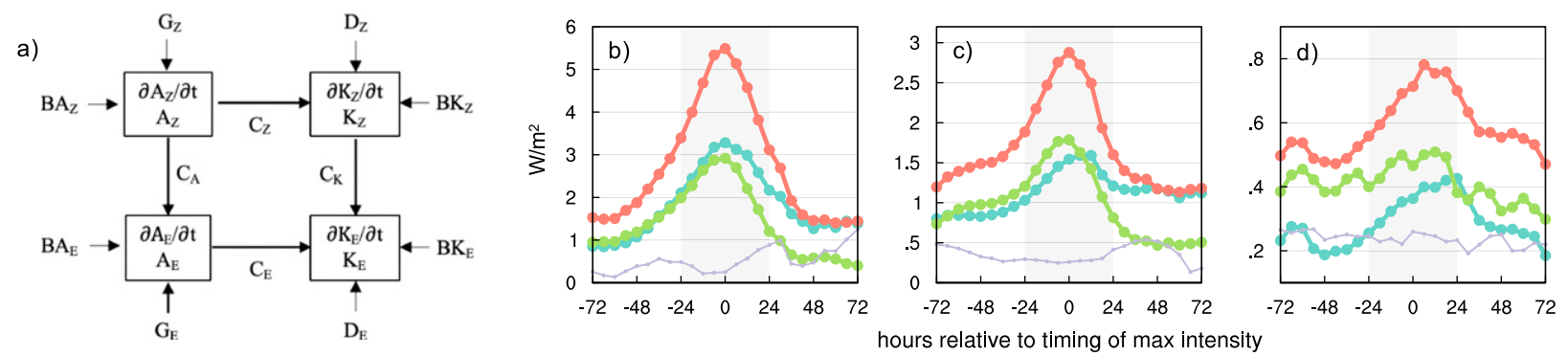

FIG. 6. (a) Complete Lorenz energy cycle and the quasi-Lagrangian composite of the Lorenz energy cycle terms with respect to each storm's maximum intensity for terrestrial LPSs in (b) cluster 1, (c) cluster 2, and (d) cluster 3 . The colored lines indicate the energy transfers from eddy available potential energy to eddy kinetic energy $C_{E}$ (red), from zonal available potential energy to eddy available potential energy $C_{A}$ (cyan), from zonal kinetic energy to eddy kinetic energy $C_{K}$ (purple), and the eddy available potential energy generation $G_{E}$ (green). Units are in $\mathrm{W} \mathrm{m}^{-2}$.

further intensifying by the weak environmental baroclinicity and are more easily subject to frictional dissipation.

We test this hypothesis in Fig. 7, which illustrates the quasiLagrangian evolution of the vertical shear of zonal wind and precipitable water, along with their climatological spatial distributions. For migratory LPSs, the environmental baroclinicity, which is reflected in vertical wind shear, slightly increases during the incipient stage but remains nearly unchanged afterward (Fig. 7a). In contrast, the precipitable water decreases dramatically along the LPSs trajectories, leading to the rapid drop-off of rainfall and $G_{E}$ (Figs. 4a-d and 7b). The temporal evolution of wind shear and precipitable water makes intuitive sense as the migratory LPSs propagate into the climatological baroclinic zone (Fig. 7d) but move out of the humid monsoon region (Fig. 7e). For the quasi-stationary LPSs, the baroclinicity is rather weak and precipitable water stays high throughout the storms' lifespans, as they tend to form and stay in a region enriched in water vapor but with weak baroclinicity. To conclude, the abundant water vapor in the East Asian monsoon region (Fig. 7e) make the area favorable for cyclogenesis and intensification in summer. Although the summertime baroclinicity is vital for the growth of terrestrial LPSs, it is not sufficient to maintain the strength of the LPSs as they dissipate rapidly after propagating into the drier extratropics. The overwhelming role of latent heat release
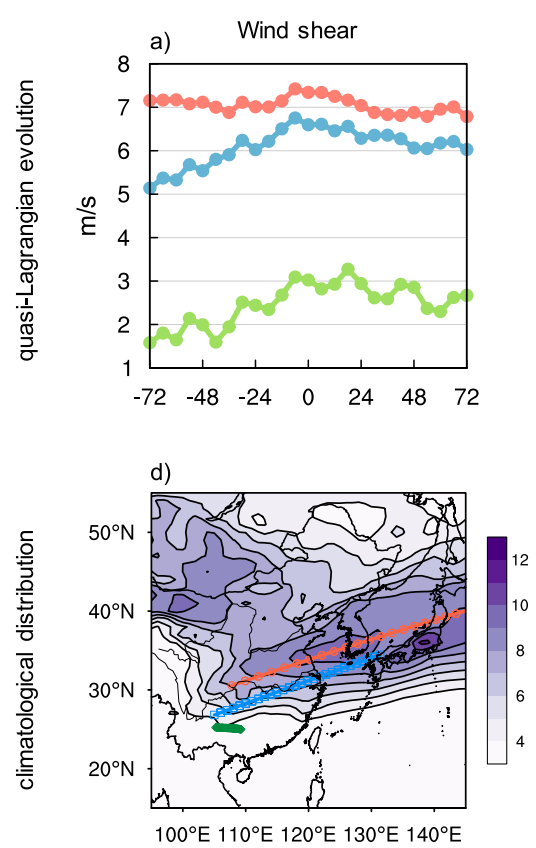

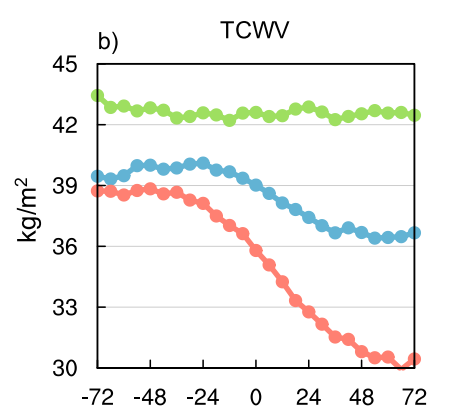

hours relative to timing of max intensity

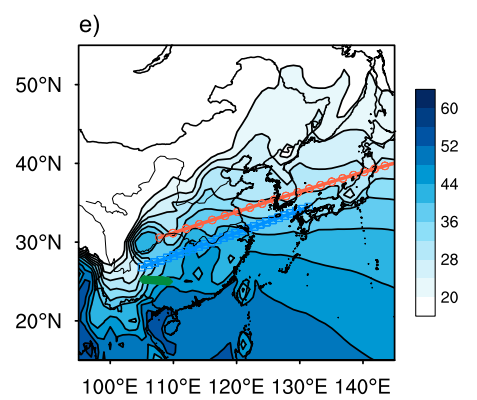

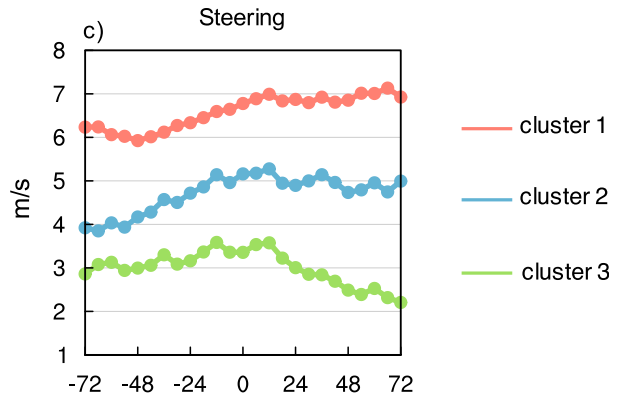

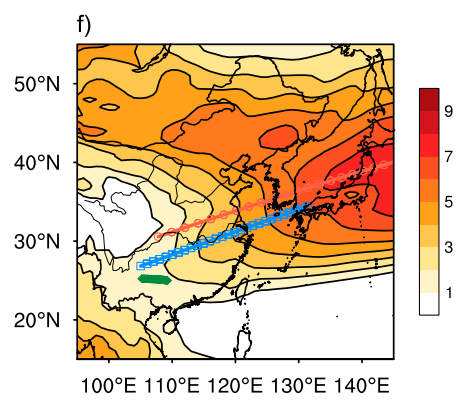

FIG. 7. (top) Quasi-Lagrangian composites with respect to each storm's maximum intensity for (a) vertical shear of zonal wind between 500 and $850 \mathrm{hPa}\left(\mathrm{m} \mathrm{s}^{-1}\right)$, (b) total column water vapor $\left(\mathrm{kg} \mathrm{m}^{-2}\right)$, and (c) mean 500-850-hPa zonal flow ( $\mathrm{m} \mathrm{s}^{-1}$ ). (bottom) The climatological summer (May-September) (d) vertical shear of zonal wind between 500 and $850 \mathrm{hPa}\left(\mathrm{m} \mathrm{s}^{-1}\right)$, (e) total column water vapor $\left(\mathrm{kg} \mathrm{m}^{-2}\right)$, and (f) mean 500-850-hPa zonal flow $\left(\mathrm{m} \mathrm{s}^{-1}\right)$ overlapped with the mean regression tracks of the three clusters. 

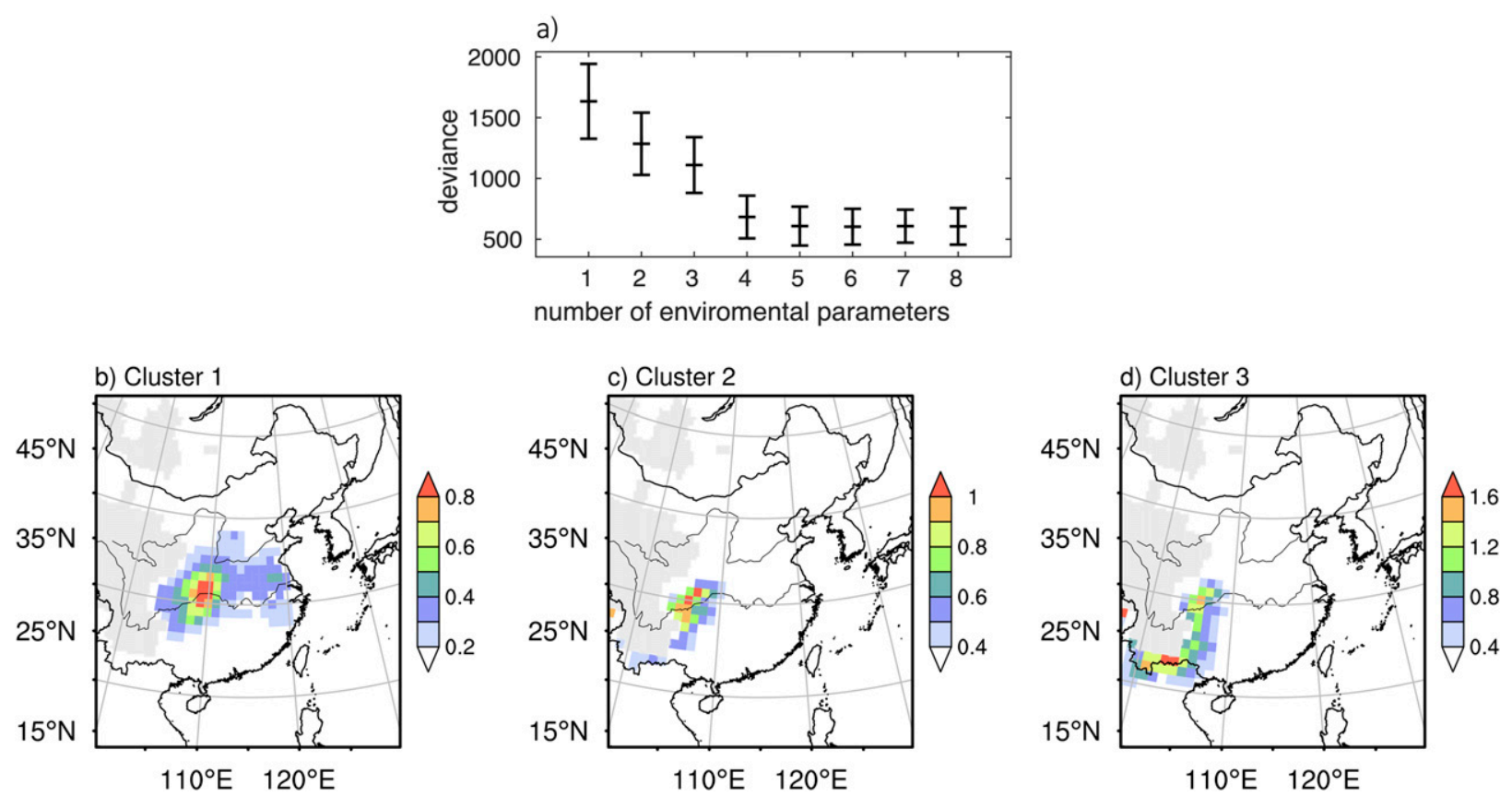

FIG. 8. (a) Mean standard deviation of the Poisson regression as a function of the number of environmental variables included. The error bars denote one standard deviation. (b)-(d) Genesis indices for the three clusters based on the Poisson regression (number of LPSs per summer).

distinguishes the terrestrial monsoon LPSs from the wintertime extratropical cyclones for which the energetics is dominated by baroclinic instability (Black and Pezza 2013).

\section{c. Environmental constraint on the trend of cluster occurrence}

As illustrated in Fig. 5, the occurrence of clusters 1 and 2 decreases, whereas the occurrence of cluster 3 increases over the study period. It is still not clear what changes in environmental conditions would favor fewer baroclinic-driven migratory cyclones and more moisture-initiated quasi-stationary cyclones. To reveal the dependence of the climatological LPS genesis distribution on the mean climatological conditions, we adopt the Poisson regression, which is a traditional modeling approach for noncontinuous variables such as the storm counts. Similar empirical genesis indices have been constructed for the climatological genesis distribution of tropical cyclones, tornados, and monsoon depressions (Camargo et al. 2014; Tippett et al. 2011, 2012; Ditchek et al. 2016; Sandeep et al. 2018). For each $1^{\circ} \times 1^{\circ}$ grid, the number of LPS genesis per summer over the 40 -yr period is assumed to follow a Poisson distribution with expected value $\mu$, which has a log-linear dependence on a vector of environmental parameters $\mathbf{x}$ modeled by

$$
\mu=\exp \left[\mathbf{b}^{\mathrm{T}} \mathbf{x}+\log (\Delta x \Delta y \cos \phi)\right] .
$$

Here $\mathbf{b}$ is the vector of regression coefficients derived using the standard maximum likelihood method; $\Delta x$ and $\Delta y$ are latitude and longitude grid spacing in degrees, respectively, and are both equal to one; and $\phi$ denotes the latitudes. The second term in the bracket denotes an offset term to make coefficient $\mathbf{b}$ valid for equal area grids.

As terrestrial LPSs are fueled by moist baroclinic instability with topographically preferred cyclogenesis on the Tibetan Plateau's downwind flank (Figs. 2-7), the candidate environmental variables that may impact LPS genesis include relative vorticity, vertical wind shear, surface temperature, atmospheric convective stability (i.e., the lapse rate of equivalent potential temperature, convective available potential energy, and convective precipitation), and atmospheric moisture content (i.e., total column water vapor, relative humidity, and specific humidity). As the clustering method divides the terrestrial LPSs into three clusters that have very different translation speed as influenced by the steering flow (Table 1; Figs. 7c,f), we further include the column-averaged wind (i.e., zonal wind, meridional wind, and total wind speed) between 500 and $850 \mathrm{hPa}$ as the steering flow strength to accommodate such differences. To avoid statistical overfitting, a subset of variables of best fitness is objectively identified using the forward sequential feature selection based on best fit (e.g., Tippett et al. 2011). The forward sequential feature selection starts from a null variable set and, in each iteration, the one remaining variable from the candidate pool that best improves the model is added. Except for column-integrated variables, the variables are sampled across multiple pressure levels and the areas falling below ground are masked out. For a fair comparison among clusters, we begin with the forward selection to determine the environmental variables that best fit the genesis of all terrestrial LPSs without considering their differences among different clusters. 

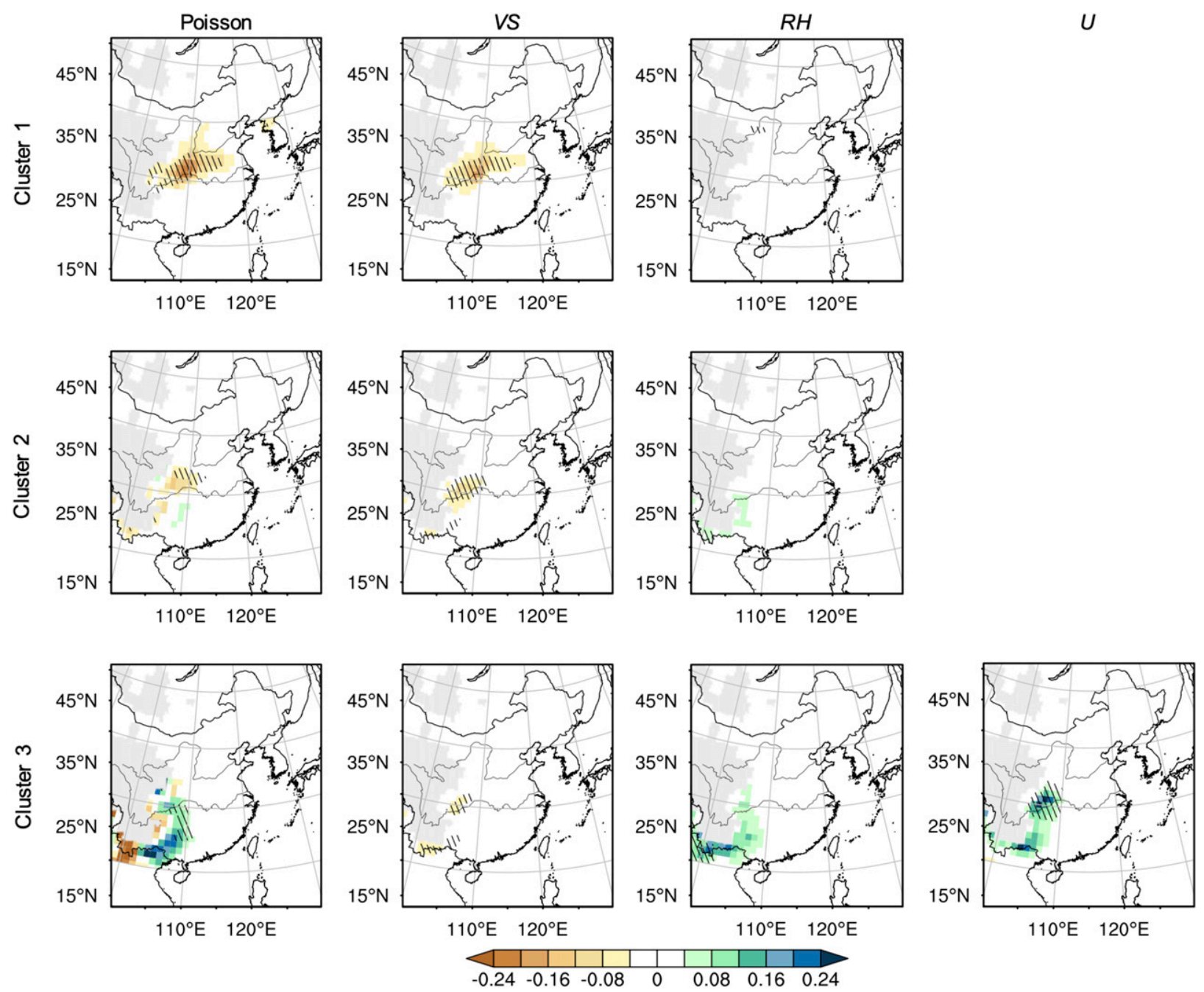

FIG. 9. Linear trend (number of LPSs per decade) of Poisson genesis indices with (first column) all varying environmental parameters, (second column) varying vertical zonal wind shear only (VS), (third column) varying relative humidity only (RH), and (fourth column) varying column-averaged zonal wind only $(U)$ for (top) cluster 1 , (middle) cluster 2 , and (bottom) cluster 3 . Stippling indicates statistical significance greater than $90 \%$ confidence level according to a nonparametric Mann-Kendall test.

Figure $8 \mathrm{a}$ presents the mean standard deviation of the Poisson regression, which is inversely proportional to the goodness of fit, as a function of the number of environmental variables included. The deviance decreases significantly as the number of environmental parameters increases from one to four. Further reduction in deviation slows significantly as more variables are added beyond the initial four. Therefore, four environmental parameters are chosen by the forward selection method, including the column-averaged relative vorticity between 500 and $850 \mathrm{hPa}\left(\xi ; 10^{-5} \mathrm{~s}^{-1}\right)$, vertical shear of zonal wind between 500 and $850 \mathrm{hPa}\left(\mathrm{VS} ; \mathrm{m} \mathrm{s}^{-1}\right)$, lapse rate of equivalent potential temperature between 500 and $850 \mathrm{hPa}$ (LR; $\left.10^{-2} \mathrm{~K} \mathrm{hPa}^{-1}\right)$, and relative humidity at $750 \mathrm{hPa}(\mathrm{RH} ; \%)$. The only exception is cluster 3 , for which including the zonal wind $U$ leads to a significant improvement of fitness. The zonal wind appears to provide a better fit than meridional wind and total wind speed probably because the propagation direction is more zonally oriented (Fig. 2h). The resulting genesis indices for the three clusters are as follows:

$$
\begin{aligned}
\text { cluster } 1: \mu_{1}= & \exp (-10.31+0.66 \mathrm{VS}+0.07 \xi \\
& +0.07 \mathrm{LR}+0.08 \mathrm{RH}), \\
\text { cluster } 2: \mu_{2}= & \exp (-10.34+0.38 \mathrm{VS}+0.75 \xi \\
& +0.40 \mathrm{LR}+0.10 \mathrm{RH}), \\
\text { cluster } 3: \mu_{3}= & \exp (-11.04+0.17 \mathrm{VS}+1.14 \xi \\
& +0.64 \mathrm{LR}+0.12 \mathrm{RH}-0.97 U) .
\end{aligned}
$$

Only those terms that significantly contribute to the genesis $(p<0.1)$ are included in the above regressions. The relative importance of these environmental factors differs among clusters. Cluster 1 is more affected by baroclinicity (VS), whereas the relative vorticity $(\xi)$, atmospheric stability (LR), and midtropospheric 
a) VS

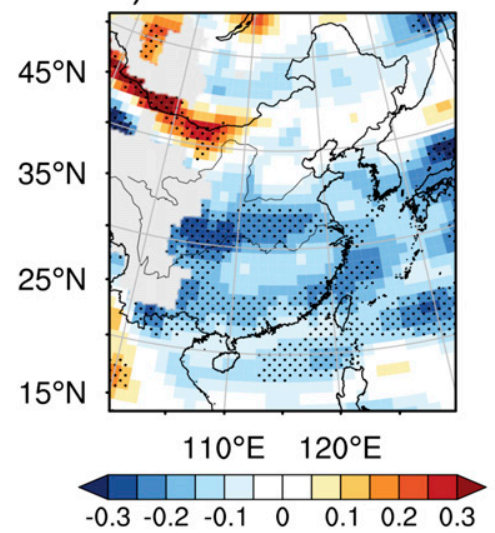

b) $\mathrm{RH}$

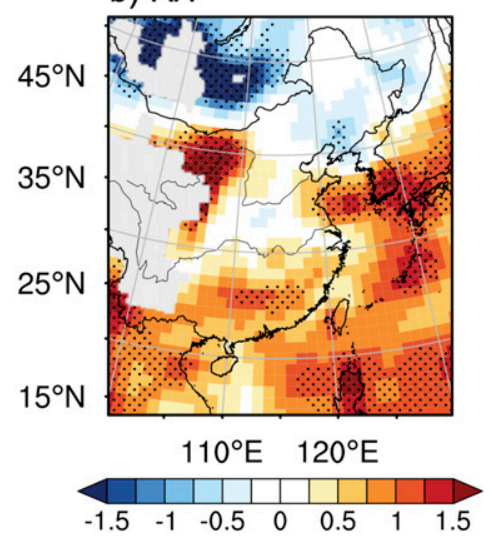

c) $U$

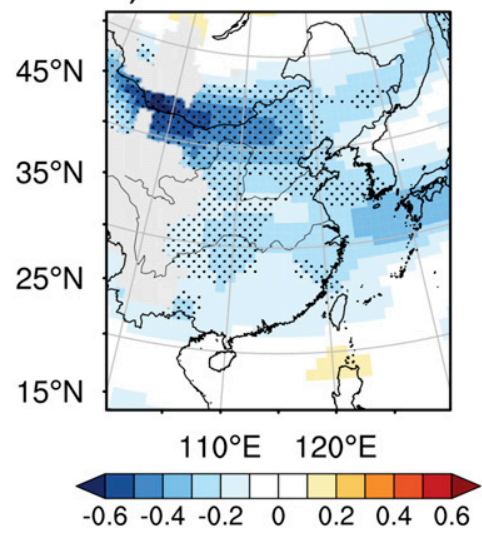

FIG. 10. Linear trend of (a) vertical zonal wind shear VS $\left(\mathrm{m} \mathrm{s}^{-1} \mathrm{decade}^{-1}\right)$, (b) relative humidity RH (\% decade $\left.{ }^{-1}\right)$, and (c) columnaveraged zonal wind $U\left(\mathrm{~m} \mathrm{~s}^{-1}\right.$ decade $\left.^{-1}\right)$. Stippling indicates statistical significance greater than the $90 \%$ confidence level according to the two-tailed Student's $t$ test.

relative humidity $(\mathrm{RH})$ are more effective in influencing the occurrence of clusters 2 and 3. Additionally, the occurrence of cluster 3 is inversely proportional to the steering flow $U$. Overall, the genesis indices of the three clusters encapsulate much of the physical processes controlling the occurrence of different types of terrestrial LPSs as discussed in earlier sections. The genesis indices derived from the above relationship are shown in Figs. 8a-d, which broadly agree with the observed genesis in Figs. 2a, 2d, and 2g, although there are some deviations in details.

Although the Poisson regression fits reasonably well with the climatological genesis distribution, it is not a guarantee that the indices can capture the observed trends shown in Fig. 3 because the spatial dependence of the LPS occurrence on environmental variables is not necessarily identical to the temporal dependence. Therefore, we test the ability of the genesis indices to capture the trends of cluster occurrence by substituting the timeindependent environmental variables in Eqs. (6)-(8) with their yearly varying values (Fig. 9). The linear trends of the three relevant environmental variables, including vertical wind shear, relative humidity, and steering wind, are plotted in Fig. 10. The time-varying genesis indices reproduce the decreasing trend of mobile LPSs and the increasing trend of quasi-stationary LPSs (Fig. 9, first column), broadly consistent with the observed trends (Figs. 5a-c). By allowing one variable to vary and keeping the remaining fixed at the climatological values, we evaluate the relative contribution of each environmental variable to the trends. The decreasing occurrence of migratory LPSs is mostly driven by the decreased baroclinic instability VS (Fig. 9, second column), consistent with the reduced vertical wind shear across the East Asian monsoon region as shown in Fig. 10a. On the other hand, the increasing occurrence of cluster 3 is associated with decreased steering flow strength $U$ and increased $\mathrm{RH}$ in the mid-to-lower troposphere over East Asia (Figs. 9 and 10b,c).

\section{Summary}

Given the significance of terrestrial LPSs in modulating the continental extreme rainfall over the East Asian summer monsoon region, we have assessed in this study the physical processes fostering the formation and intensification of the different types of terrestrial LPSs and explored the environmental factors regulating their temporal trends. The trajectories of terrestrial LPSs in the period of 1979-2018 were objectively partitioned into three clusters using a probabilistic clustering method. Prominent intercluster differences were identified regarding the LPS tracks, temporal evolution, energetics, and trends in storm numbers. The difference in the tracks distinguishes the clusters into two regimes: a migratory type and a quasi-stationary one. The migratory LPSs in clusters 1 and 2, which propagate northeastward toward the subtropical jet, exhibit a substantial growth rate accompanied by intense rainfall before reaching their lifetime maximum intensity near the East Asian coasts. Subsequently, the LPS rainfall decreases sharply, and storms dissipate quickly. In comparison, for the quasi-stationary cluster 3, which forms closer to the Tibetan Plateau and moves slowly, the growth rate is comparatively moderate, and the lifetime maximum intensity occurs simultaneously with the peak rainfall rate, suggesting that the interactions between latent heat release and storm circulation are dominant.

On the basis of the limited-area Lorenz energy cycle, we have demonstrated that the condensational heating and the baroclinic instability are indispensable energy sources for the development of terrestrial LPSs. However, the relative importance of the two energy sources differs among clusters and cyclone development stages. In the developing stage of the migratory LPSs, the two energy sources are of equal importance. Whereas in the decaying stage, even though the storms are located much closer to the subtropical jet with stronger environmental baroclinicity, they are not able to sustain their intensity without a sustained latent heat release. The rapid decline of latent heat release is related to the substantial dropoff of total precipitable water, as the LPSs move out of the moisture-enriched monsoon region. In contrast, for the weaker quasi-stationary LPSs in cluster 3, the latent heat release dominates over baroclinicity throughout the life cycle as these 
LPSs form and stay in the water vapor enriched region with relatively weak baroclinicity. These results suggest that the summer baroclinicity is vital for the amplification of terrestrial LPSs. However, baroclinicity alone is not enough to maintain the storms as they dissipate rapidly after propagating into the drier extratropics. The enriched water vapor sets the East Asian monsoon region as an area favorable for summer cyclogenesis.

Over the study period, the number of migratory LPSs decreases but the number of quasi-stationary LPSs increases, thereby contributing to the "south flood-north drought" rainfall trends over eastern China (e.g., Zhai et al. 2005; Liu et al. 2005; You and Ting 2021). Based on the aforementioned energetics analysis and a Poisson regression model, we have developed empirical genesis indices that successfully associate the climatological LPS genesis distribution with the local environmental conditions and reproduce the observed trends of LPS occurrence. Additionally, the genesis indices indicate that the declining number of migratory LPSs is related to the reduced baroclinicity, while the increasing occurrence of quasi-stationary LPSs is caused by the enhanced relative humidity and the reduced steering flow in the mid-to-lower troposphere over East Asia. Future changes of the terrestrial LPSs will depend on how the environmental factors vary as a response to the anthropogenic forcings, which will be explored in future studies using climate model simulations.

Acknowledgments. We would like to acknowledge the highperformance computing support from the High-Performance Computing Service at Columbia University, sponsored by the Columbia Center for Climate and Life. This research was supported by the National Science Foundation Grant AGS16-07348. We thank three anonymous reviewers for their constructive and valuable suggestions. Y. You. was supported by National Aeronautics and Space Administration (NASA) under the Future Investigators in NASA Earth and Space Science and Technology (FINESST) program-Grant 19-EARTH20-0243.

\section{APPENDIX}

\section{Mathematical Expressions of Components in the Limited-Area Lorenz Energy Cycle}

In the mathematical expressions of Lorenz energy cycle, $[X]_{\lambda}$ represents the zonal mean of variable $X$ between longitude $\lambda_{1}$ and $\lambda_{1}$,

$$
[X]_{\lambda}=\frac{1}{\lambda_{2}-\lambda_{1}} \int_{p_{1}}^{p_{2}} X d \lambda
$$

$(X)_{\lambda}$ denotes the eddy component of variable $X$,

$$
(X)_{\lambda}=X-[X]_{\lambda}
$$

$[X]_{\lambda \phi}$ is the average of $X$ in area bounded by longitude $\lambda_{1}$ and $\lambda_{2}$ and latitude $\phi_{1}$ and $\phi_{2}$,

$$
[X]_{\lambda \phi}=\frac{1}{\lambda_{2}-\lambda_{1}} \frac{1}{\sin \phi_{2}-\sin \phi_{1}} \int_{\phi_{1}}^{\phi_{2}} \int_{\lambda_{1}}^{\lambda_{2}} X \cos \phi d \lambda d \phi .
$$

The energy conversion terms examined in the present study can be written as

$$
\begin{aligned}
& C_{K}=-\left\{\int_{p_{1}}^{p_{2}} \frac{1}{g}\left[(v)_{\lambda}(u)_{\lambda} \frac{\cos \phi}{r} \frac{\partial}{\partial \phi}\left(\frac{[u]_{\lambda}}{\cos \phi}\right)\right]_{\lambda \phi}\right. \\
& +\int_{p_{1}}^{p_{2}} \frac{1}{g}\left[\frac{(v)_{\lambda}^{2}}{r} \frac{\partial[v]_{\lambda}}{\partial \phi}\right]_{\lambda \phi}+\int_{p_{1}}^{p_{2}} \frac{1}{g}\left[[v]_{\lambda}(u)_{\lambda}^{2} \frac{\tan \phi}{r}\right]_{\lambda \phi} \\
& \left.+\int_{p_{1}}^{p_{2}} \frac{1}{g}\left[(\omega)_{\lambda}(u)_{\lambda} \frac{\partial[u]_{\lambda}}{\partial p}\right]_{\lambda \phi}+\int_{p_{1}}^{p_{2}} \frac{1}{g}\left[(\omega)_{\lambda}(v)_{\lambda} \frac{\partial[v]_{\lambda}}{\partial p}\right]_{\lambda \phi}\right\} d p, \\
& C_{E}=\int_{p_{1}}^{p_{2}}-\left[(\omega)_{\lambda}(T)_{\lambda}\right] \underset{\lambda \phi}{\frac{R}{g p}} d p, \\
& C_{A}=\int_{p_{2}}^{p_{2}}-\left\{\left[\frac{(v)_{\lambda}(T)_{\lambda}}{2[\sigma]_{\lambda \phi} r} \frac{\partial\left([T]_{\lambda}\right)}{\partial \phi}\right]_{\lambda \phi}\right. \\
& \left.+\left[\frac{(\omega)_{\lambda}(T)_{\lambda}}{p^{R / c_{p}}} \times \frac{\partial}{\partial p}\left(\frac{\left([T]_{\lambda}\right)_{\phi} p^{R / c_{p}}}{[\sigma]_{\lambda \phi}}\right)\right]_{\lambda \phi}\right\} d p, \\
& G_{E}=\int_{p_{1}}^{p_{2}} \frac{\left[(q)_{\lambda}[T]_{\lambda}\right]_{\lambda \phi}}{c_{p}[\sigma]_{\lambda \phi}} d p
\end{aligned}
$$

in which $\sigma$ is the static stability parameter, which is given by

$$
[\sigma]_{\lambda \phi}=\left[\frac{g T}{c_{p}}-\frac{g p}{R} \frac{\partial T}{\partial p}\right]_{\lambda \phi}
$$

where $u$ and $v$ represent the zonal and meridional wind, respectively, and $w$ is the vertical velocity in pressure coordinates; $R$ is the gas constant law, $g$ is the magnitude of acceleration of gravity, $T$ is air temperature, $r$ is the radius of Earth, $c_{p}$ is specific heat capacity at constant pressure, and $q$ is diabatic heating.

\section{REFERENCES}

Black, M. T., and A. B. Pezza, 2013: A universal, broad- environment energy conversion signature of explosive cyclones. Geophys. Res. Lett., 40, 452-457, https://doi.org/10.1002/grl.50114.

Bolton, D., 1980: The computation of equivalent potential temperature. Mon. Wea. Rev., 108, 1046-1053, https://doi.org/ 10.1175/1520-0493(1980)108<1046:TCOEPT > 2.0.CO;2.

Camargo, S. J., A. W. Robertson, S. J. Gaffney, P. Smyth, and M. Ghil, 2007a: Cluster analysis of typhoon tracks: Part I: General properties. J. Climate, 20, 3635-3653, https://doi.org/ 10.1175/JCLI4188.1.

,,,,---- , and $-2007 \mathrm{~b}$ : Cluster analysis of typhoon tracks: Part II: Large-scale circulation and ENSO. J. Climate, 20, 3654-3676, https://doi.org/10.1175/JCLI4203.1.

- — - A. G. Barnston, and M. Ghil, 2008: Clustering of eastern North Pacific tropical cyclone tracks: ENSO and MJO effects. Geochem. Geophys. Geosyst., 9, Q06V05, https:// doi.org/10.1029/2007GC001861.

, M. K. Tippett, A. H. Sobel, G. A. Vecchi, and M. Zhao, 2014: Testing the performance of tropical cyclone genesis indices in 
future climates using the HIRAM model. J. Climate, 27, 91719196, https://doi.org/10.1175/JCLI-D-13-00505.1.

Chan, S. C., and S. Nigam, 2009: Residual diagnosis of diabatic heating from ERA-40 and NCEP reanalyses: Intercomparisons with TRMM. J. Climate, 22, 414-428, https://doi.org/10.1175/ 2008JCLI2417.1.

Chang, C.-P., L. Yan, and G. T.-J. Chen, 2000: A numerical simulation of vortex development during the 1992 East Asian summer monsoon onset using the navy's regional model. Mon. Wea. Rev., 128, 1604-1631, https://doi.org/10.1175/ 1520-0493(2000)128<1604:ANSOVD>2.0.CO;2.

— - Y. Lei, C.-H. Sui, X. Lin, and F. Ren, 2012: Tropical cyclone and extreme rainfall trends in East Asian summer monsoon since mid-20th century. Geophys. Res. Lett., 39, L18702, https://doi.org/10.1029/2012GL052945.

Chen, Y. R., Y. Q. Li, and T. L. Zhao, 2015: Cause analysis on eastward movement of southwest China vortex and its induced heavy rainfall in South China. Adv. Meteor., 2015, 481735, https://doi.org/10.1155/2015/481735.

Cohen, N. Y., and W. R. Boos, 2016: Perspectives on moist baroclinic instability: Implications for the growth of monsoon depressions. J. Atmos. Sci., 73, 1767-1788, https://doi.org/10.1175/ JAS-D-15-0254.1.

Curio, J., R. Schiemann, K. I. Hodges, and A. G. Turner, 2019: Climatology of Tibetan Plateau vortices in reanalysis data and a high-resolution global climate model. J. Climate, 32, 1933-1950, https://doi.org/10.1175/JCLI-D-18-0021.1

Daloz, A. S., and Coauthors, 2015: Cluster analysis of explicitly and downscaled simulated North Atlantic tropical cyclone tracks. J. Climate, 28, 1333-1361, https://doi.org/10.1175/JCLI-D-1300646.1 .

Day, J. A., I. Fung, and W. Liu, 2018: Changing character of rainfall in eastern China, 1951-2007. Proc. Natl. Acad. Sci. USA, 115, 2016-2021, https://doi.org/10.1073/pnas. 1715386115.

Dee, D. P., and Coauthors, 2011: The ERA-Interim reanalysis: Configuration and performance of the data assimilation system. Quart. J. Roy. Meteor. Soc., 137, 553-597, https://doi.org/ 10.1002/qj.828.

Ditchek, S. D., W. R. Boos, S. J. Camargo, and M. K. Tippett, 2016: A genesis index for monsoon disturbances. J. Climate, 29, 5189-5203, https://doi.org/10.1175/JCLI-D-15-0704.1.

Dong, Q., X. Chen, and T. X. Chen, 2011: Characteristics and changes of extreme precipitation in the Yellow-Huaihe and Yangtze-Huaihe Rivers basins, China. J. Climate, 24, 37813795, https://doi.org/10.1175/2010JCLI3653.1.

Feng, X.-Y., C.-H. Liu, G.-Z. Fan, X. D. Liu, and C. Y. Feng, 2016: Climatology and structures of southwest vortices in the NCEP Climate Forecast System Reanalysis. J. Climate, 29, 76757701, https://doi.org/10.1175/JCLI-D-15-0813.1.

Gaffney, S. J., 2004: Probabilistic curve-aligned clustering and prediction with regression mixture models. Ph.D. thesis, University of California, 281 pp., https:/www.ics.uci.edu/pub/ sgaffney/outgoing/sgaffney_thesis.pdf.

— A. W. Robertson, P. Smyth, S. J. Camargo, and M. Ghil, 2007: Probabilistic clustering of extratropical cyclones using regression mixture models. Climate Dyn., 29, 423-440, https:// doi.org/10.1007/s00382-007-0235-z.

Guo, L., N. P. Klingaman, P. L. Vidale, A. G. Turner, M.-E. Demory, and A. Cobb, 2017: Contribution of tropical cyclones to atmospheric moisture transport and rainfall over East Asia. J. Climate, 30, 3853-3865, https://doi.org/10.1175/ JCLI-D-16-0308.1.
Gutowski, W. J., Jr., L. E. Branscome, and D. A. Stewart, 1992: Life cycles of moist baroclinic eddies. J. Atmos. Sci., 49, 306-319, https://doi.org/10.1175/1520-0469(1992)049<0306: LCOMBE $>2.0 . \mathrm{CO} ; 2$.

Hodges, K. I., 1994: A general method for tracking analysis and its application to meteorological data. Mon. Wea. Rev., 122, 2573-2586, https://doi.org/10.1175/1520-0493(1994)122<2573: AGMFTA $>2.0$.CO.

- 1999: Adaptive constraints for feature tracking. Mon. Wea. Rev., 127, 1362-1373, https://doi.org/10.1175/1520-0493(1999) $127<1362$ :ACFFT $>2.0$. CO 2 .

Hurley, J. V., and W. R. Boos, 2015: A global climatology of monsoon low-pressure systems. Quart. J. Roy. Meteor. Soc., 141, 1049-1064, doi:10.1002/qj.2447.

Khouakhi, A., G. Villarini, and G. A. Vecchi, 2017: Contribution of tropical cyclones to rainfall at the global scale. J. Climate, 30, 359-372, https://doi.org/10.1175/JCLI-D-16-0298.1.

Kossin, J. P., S. J. Camargo, and M. Sitkowski, 2010: Climate modulation of North Atlantic hurricane tracks. J. Climate, 23, 3057-3076, https://doi.org/10.1175/2010JCLI3497.1.

Krishnamurti, T. N., A. Martin, R. Krishnamurti, A. Simon, A. Thomas, and V. Kumar, 2013: Impacts of enhanced CCN on the organization of convection and recent reduced counts of monsoon depressions. Climate Dyn., 41, 117-134, https:// doi.org/10.1007/s00382-012-1638-z.

Kuo, Y.-H., L. Cheng, and R. A. Anthes, 1986: Mesoscale analyses of the Sichuan flood catastrophe, 11-15 July 1981. Mon. Wea. Rev., 114, 1984-2003, https://doi.org/10.1175/1520-0493(1986) $114<1984$ :MAOTSF $>2.0$. CO;2.

Li, R. C. Y., W. Zhou, and T. C. Lee, 2015: Climatological characteristics and observed trends of tropical cyclone-induced rainfall and their influences on long-term rainfall variations in Hong Kong. Mon. Wea. Rev., 143, 2192-2206, https://doi.org/ 10.1175/MWR-D-14-00332.1.

Liu, B., M. Xu, M. Henderson, and Y. Qi, 2005: Observed trends of precipitation amount, frequency, and intensity in China, 19962000. J. Geophys. Res., 110, D08103, https://doi.org/10.1029/ 2004JD004864.

Lorenz, E. N., 1955: Available potential energy and the maintenance of the general circulation. Tellus, 7, 157-167, https:// doi.org/10.3402/tellusa.v7i2.8796.

Lu, J. H., 1986: Introduction to the Southwest Vortex. China Meteorological Press, $270 \mathrm{pp}$.

Ma, S., and Coauthors, 2017: Detectable anthropogenic shift toward heavy precipitation over eastern China. J. Climate, 30, 1381-1396, https://doi.org/10.1175/JCLI-D-16-0311.1.

Mak, M., 1982: On moist quasi-geostrophic baroclinic instability. J. Atmos. Sci., 39, 2028-2037, https://doi.org/10.1175/15200469(1982)039<2028:OMQGBI >2.0.CO;2.

Michaelides, S. C., 1987: Limited area energetics of Genoa cyclogenesis. Mon. Wea. Rev., 115, 13-26, https://doi.org/10.1175/ 1520-0493(1987)115<0013:LAEOGC > 2.0.CO;2.

, 1992: A spatial and temporal energetics analysis of a baroclinic disturbance in the Mediterranean. Mon. Wea. Rev., 120, 1224-1243, https:// doi.org/10.1175/1520-0493(1992)120<1224:ASATEA>2.0.CO;2.

_, N. G. Prezerakos, and H. A. Flocas, 1999: Quasi-Lagrangian energetics of an intense Mediterranean cyclone. Quart. J. Roy. Meteor. Soc., 125, 139-168, https://doi.org/10.1002/ qj.49712555309.

Nakamura, J., and Coauthors, 2017: Western North Pacific tropical cyclone model tracks in present and future climates. J. Geophys. Res. Atmos., 122, 9721-9744, https://doi.org/10.1002/ 2017JD027007. 
Patricola, C. M., S. J. Camargo, P. J. Klotzbach, R. Saravanan, and P. Chang, 2018: The influence of ENSO flavors on western North Pacific tropical cyclone activity. J. Climate, 31, 53955416, https://doi.org/10.1175/JCLI-D-17-0678.1.

Ramsay, H. A., S. J. Camargo, and D. Kim, 2012: Cluster analysis of tropical cyclone tracks in the Southern Hemisphere. Climate Dyn., 39, 897-917, https://doi.org/10.1007/s00382-0111225-8.

_ - S. S. Chand, and S. J. Camargo, 2018: A statistical assessment of Southern Hemisphere tropical cyclone tracks in climate models. J. Climate, 31, 10 081-10 104, https://doi.org/10.1175/ JCLI-D-18-0377.1.

Sandeep, S., R. S. Ajayamohan, W. R. Boos, T. P. Sabin, and V. Praveen, 2018: Decline and poleward shift in Indian summer monsoon synoptic activity in a warming climate. Proc. Natl. Acad. Sci. USA, 115, 2681-2686, https://doi.org/10.1073/ pnas. 1709031115.

Tao, S.-Y., and Y.-H. Ding, 1981: Observational evidence of the influence of the Qinghai Xizang (Tibet) Plateau on the occurrence of heavy rain and severe convective storms in China. Bull. Amer. Meteor. Soc., 62, 23-30, https://doi.org/10.1175/ 1520-0477(1981)062<0023:OEOTIO > 2.0.CO;2.

Tippett, M. K., S. J. Camargo, and A. H. Sobel, 2011: A Poisson regression index for tropical cyclone genesis and the role of large-scale vorticity in genesis. J. Climate, 24, 2335-2357, https://doi.org/10.1175/2010JCLI3811.1.

__ A. H. Sobel, and S. J. Camargo, 2012: Association of U.S. tornado occurrence with monthly environmental parameters. Geophys. Res. Lett., 39, L02801, https://doi.org/10.1029/ 2011 GL050368.
Veiga, J. A. P., and T. Ambrizzi, 2013: A global and hemispherical analysis of the Lorenz energetics based on the representative concentration pathways used in CMIP5. Adv. Meteor., 2013, 485047, https://doi.org/10.1155/2013/485047.

Wang, B., and I. Orlanski, 1987: Study of a heavy rain vortex formed over the eastern flank of the Tibetan Plateau. Mon. Wea. Rev., 115, 1370-1393, https://doi.org/10.1175/1520-0493(1987) $115<1370:$ SOAHRV $>2.0 . C O ; 2$.

Wang, W., Y.-H. Kuo, and T. T. Warner, 1993: A diabatically driven mesoscale vortex in the lee of the Tibetan Plateau. Mon. Wea. Rev., 121, 2542-2561, https://doi.org/10.1175/15200493(1993)121<2542:ADDMVI>2.0.CO;2.

Wang, Y., and L. Zhou, 2005: Observed trends in extreme precipitation events in China during 1961-2001 and the associated changes in large-scale circulation. Geophys. Res. Lett., 32, L09707, https://doi.org/10.1029/2005GL023769.

You, Y., and M. Ting, 2021: Low pressure systems and extreme precipitation in Southeast and East Asian monsoon regions. J. Climate, 34, 1147-1162, https://doi.org/10.1175/JCLI-D-20-0206.1.

Zhai, P. M., X. B. Zhang, H. Wan, and X. H. Pan, 2005: Trends in total precipitation and frequency of daily precipitation extremes over China. J. Climate, 18, 1096-1108, https://doi.org/ 10.1175/JCLI-3318.1.

Zhang, F., and S. Zhao, 2004: A study of formation and development of one kind of cyclone on the mei-yu (baiu) front. $A d v$. Atmos. Sci., 21, 741-754, https://doi.org/10.1007/BF02916371.

Zhang, Q., X. Gu, J. Li, P. Shi, and V. P. Singh, 2018: The impact of tropical cyclones on extreme precipitation over coastal and inland areas of China and its association to ENSO. J. Climate, 31, 1865-1880, https://doi.org/10.1175/JCLI-D-17-0474.1. 